\title{
A sweet blow for cancer cells
}

An emerging anticancer strategy known as synthetic lethal screening involves targeting tumour cells containing particular mutations with small molecules that are only cytotoxic to such cells and leave normal cells unharmed. In a study published in Science Translational Medicine, Chan and colleagues made use of von Hippel-Lindau tumour suppressor ( $V H L)$-deficient renal cell carcinoma (RCC) cells to carry out a highthroughput screen for compounds that function in a synthetic lethal manner. They show that inhibiting glucose transporter 1 (GLUT1) could be an effective approach for selectively targeting cells containing VHL mutations.

VHL is inactivated in approximately $80 \%$ of RCCs, leading to an increase in the half-life of hypoxia-inducible factor (HIF) and an upregulation of genes involved in cancer cell metabolism, which is characterized by an increased dependence on glucose uptake for glycolysis and energy production. Among the 64,000 small molecules that were screened, the authors identified a class of compounds (the 3-series) that specifically kill cells lacking VHL.

STF-31, a compound from this series, triggers necrotic cell death in cells bearing VHL mutations, in a concentration-dependent manner. Although the upregulation of HIF in $V H L$-deficient cells increases their sensitivity to STF-31, the compound's mechanism of action involves a decrease in glycolysis.
Using a fluorescent and radioactive glucose derivative, the authors showed that STF-31 inhibits glucose uptake in VHL-deficient cells by specifically binding to the central pore of GLUT1, a glucose transporter that is highly upregulated in these cells. Interestingly, inhibiting glycolysis with a non-degradable glucose analogue or with a nonspecific glucose transport inhibitor did not confer differential toxicity to RCC cells (with and without $V H L$ ), and only genetic inhibition of GLUT1 mimicked the selective cytotoxicity of STF- 31 .

To examine the effects of STF-31 in vivo, mice with $V H L$-deficient RCC xenografts were treated with the compound for up to 14 days. The marked delay in tumour growth (without toxicity) in STF-31-treated mice, compared with mice treated with vehicle alone, warrants further investigation into this series of compounds. Furthermore, disrupting glucose uptake may be a useful therapeutic strategy for treating other types of cancer in which GLUT1 is upregulated, and the use of positron emission tomography imaging will be highly useful in assessing efficacy in Phase I clinical trials.

$$
\text { Monica Hoyos Flight }
$$

ORIGINAL RESEARCH PAPER Chan, D. A. et al. Targeting GLUT1 and the Warburg effect in renal cell carcinoma by chemical synthetic lethality. Sci. Transl. Med. 3, 94 ra70 (2011) FURTHER READING Chan, D. A. \& Giaccia, A. J. Harnessing synthetic lethal interactions in anticancer drug discovery. Nature Rev. Drug Discov. 10,351-364, 2011 